\title{
An In Vitro Comparative Evaluation of the Effects of Different Bleaching Agents on Marginal Integrity of Two Different Restorative Resin Materials: An Analysis by Confocal Laser Microscopy
}

\author{
Nitesh Goyal ${ }^{1}$ Yogesh Kumar $^{2}$ Neetu Jindal ${ }^{2} \quad$ Renu Aggarwal ${ }^{2}$ \\ ${ }^{1}$ Department of Conservative and Endodontics, RUHS College of \\ Dental Science, Ganganagar, Jaipur, Rajasthan, India \\ 2Department of Conservative Dentistry and Endodontics, Surendera \\ Dental College and Research Institute, Sri Ganganagar, Rajasthan, \\ India
}

Dent J Adv Stud 2019;7:114-122

\begin{abstract}
Address for correspondence Nitesh Goyal, MDS, 194 G Block, Near Sukharia Circle, Sri Ganganagar 335001, Rajasthan, India (e-mail: n.k.g.005@gmail.com).
\end{abstract}

\begin{abstract}
Introduction In our society, with the advances in cosmetic consciousness, wellaligned, properly contoured, white and clean teeth are a symbol of health and standard of beauty. Thus, tooth bleaching has become popular cosmetic therapy among patients and dentists. Microleakage is a major challenge to the success rate for all type of restorations.

Materials and Methods Eighty maxillary human molars were collected and evaluated and Class V cavities $5 \times 3 \times 2 \mathrm{~mm}$ size were prepared with straight carbide bur (SS White) on the buccal and palatal surfaces at the cementoenamel junction of all the samples, so that the upper margin would be in enamel and lower margins on root surface. In total, 160 class $V$ cavities were prepared on maxillary molars. Then samples were randomly divided into 3 groups (microfilled and nanohybrid composite resins and control group) and 3 subgroups (Pola Office, Whiteness HP Blue, Whiteness Perfect) according to the type of composite resins and bleaching agents used. After application of bleaching agents, all samples were immersed in a contrast solution of rhodamine B fluorescent dye for 24 hours. Then using a diamond disc samples were sectioned buccolingually through the center of the restorations and sectioned teeth were evaluated for marginal microleakage under confocal laser scanning microscope

Keywords

- Ca containing

bleaching

- rhodamine B

- microleakage to check the extent of dye penetration.

Results When all composite resin groups were compared with all the bleaching agents, at occlusal and gingival levels, lowest marginal leakage scores were obtained with the microfilled composite resin bleached with Whiteness HP blue, and in nanohybrid composite lowest microleakage score were obtained with Pola office bleach at both levels.
\end{abstract}

\section{Introduction}

In our society, with the advances in cosmetic consciousness, well-aligned, properly contoured, white and clean teeth are a symbol of health and standard of beauty. ${ }^{1}$ Dental bleaching agent is a conservative alternative and minimally invasive procedure compared with direct restorations or ceramic veneers. ${ }^{2}$
Most of the time patients undergoing dental bleaching treatment have teeth with restorations, and the tooth-bleaching mechanism involves chemical processes, which could change the physical and mechanical properties of both dental tissue and restorative materials. ${ }^{3}$

So the effect of bleaching agents on dental restorative materials had been reviewed recently in few years. Chemical published online January 20, 2020
C2019 Bhojia Dental College and Hospital affiliated to Himachal Pradesh University
License terms

() (1) $\odot \circledast$ 
softening from bleaching may affect the clinical longevity of the composite restorations. ${ }^{4}$ Existing literature reveals that bleaching therapies may have a negative effect on physical properties, color, enamel and dentin bond strength, and marginal integrity of restorative materials. Bleaching agents increases the surface roughness of composite resins and decrease microhardness of composite and tooth surface. Bleaching agents can remove the outer staining from composite resins. ${ }^{5}$

Microleakage is a major challenge to the success rate for all type of restorations. ${ }^{1}$ That is why to overcome these drawbacks some bleaching agents have calcium in their composition which is added to minimize mineral loss of the tooth during the dental bleaching procedure. ${ }^{3}$

Thus, the aim of present study was to evaluate marginal integrity at tooth/composite restoration interface after application of bleaching agents

\section{Materials and Methods}

Eighty extracted maxillary human molars were used in this study ( $\boldsymbol{- \text { Fig. }} \mathbf{1}$ ). The teeth were observed under stereomicroscope to visualize the absence of crack or fractures on enamel, previous restorations, calculus, and anatomical abnormalities.

Class $\mathrm{V}$ cavities $5 \times 3 \times 2 \mathrm{~mm}$ size were prepared with straight carbide bur (SS White, New Jersey, United States) on the buccal and palatal surfaces at the cementoenamel junction of all the samples ( $\mathbf{- F i g . 2}$ ). Then total 160 class $\mathrm{V}$ cavities were prepared on maxillary molars. One hundred sixty samples were randomly divided into 3 groups and 3 subgroups according to the type of composite resins and bleaching agents used.

For the restorative procedure, each cavity was etched with 37\% phosphoric acid (Action Gel, Prevest Denpro, India) for $\sim 15$ seconds. The cavities were washed with water spray for 15 seconds, and the excess water was removed with absorbent paper and air dried. Next, the two-step etch-and-rinse adhesive (Prime and BondNT, Dentsply Sirona, Germany) was applied in two layers, according to the manufacturer's recommendation, and each layer was vigorously scrubbed in the

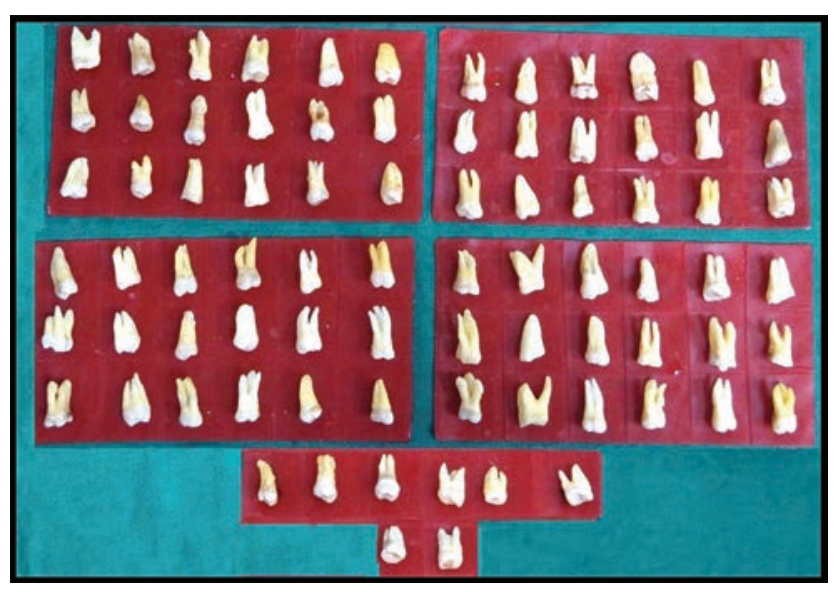

Fig. 1 Maxillary molars (80).

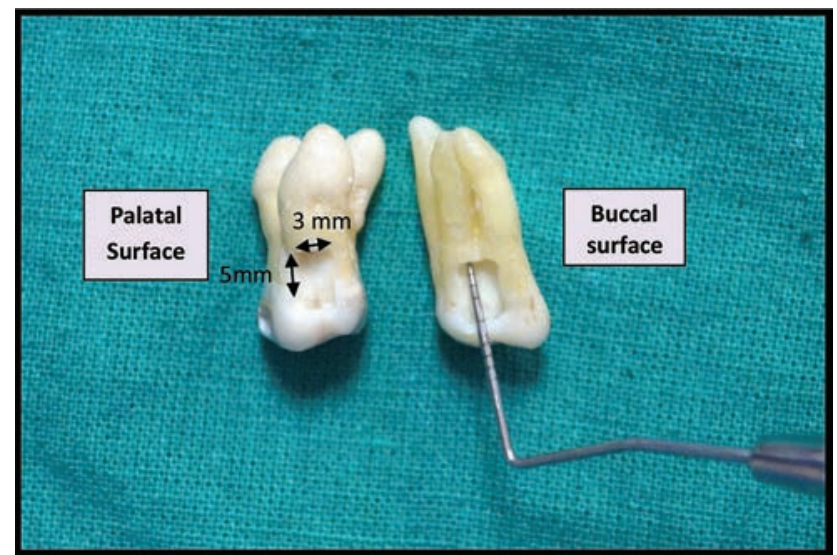

Fig. 2 Cavity Preparation $(5 \times 3 \times 2 \mathrm{~mm})$ both on buccal and palatal sides of maxillary molar.

cavity with applicator brushes for 15 seconds, permitting the solvent to evaporate. The adhesive excess was removed using a dry brush, and then the adhesive was light cured for $20 \mathrm{sec}-$ onds (LED AutoFocus 2, Satelec Acteon).

\section{Grouping of Samples}

One hundred sixty samples were randomly divided into 3 groups and 3 subgroups according to the type of composite resins and bleaching agents used.

Group I. Microfilled composite resin $(\boldsymbol{n}=\mathbf{3 0})$ : The composite resin (Heliomolar, Ivoclar Vivadent) was inserted (increments) with Teflon coated instrument (GDC) into the prepared cavities and light-cured for 30 second. Finishing and polishing of cavities was done with Sof-Lex discs (3M ESPE, United States).

Isolate the teeth with two coats of cosmetic nail polish $2 \mathrm{~mm}$ short of all the margins of each restoration before applying the bleaching agents. After that the root apices of 20 samples were mounted in acrylic resin and 10 samples for home bleaching were mounted in one arch and teeth were stored in distal water till further experiment.

Group I was further subdivided into 3 subgroups, containing 10 samples each.

Subgroup Ia: $(n=10) 35 \%$ hydrogen peroxide (Pola Office, SDI, Australia)

Teeth were cleaned with a flour-based pumice. The contents of the syringe were carefully extruded into the pot and immediately mixed using a brush applicator until homogeneous gel was achieved. A thick layer of gel was applied to all the samples and left for 8 minutes. The three applications of 8 minutes each were done. After that buccal and palatal surfaces were washed with water and air dried.

Subgroup Ib: $(n=10) 35 \%$ hydrogen peroxide with calcium (Whiteness HP Blue, FGM, Joinville, Brazil)

Teeth were cleaned with a flour-based pumice. To prepare the homogeneous gel, connecting the two syringes each other and plungers were pushed four times on each side. This homogenous mix was performed into one syringe, and applied a thick layer $(0.5-1 \mathrm{~mm})$ on the surface of samples and left for 40 minutes. With a disposable micro applicator the gel was moved on the samples frequently in every 5 to 
10 minutes to release oxygen bubbles generated and renew contact of the gel with the tooth structure. In the end of the treatment, the samples were washed with plenty of water and were polished with polishing paste.

Subgroup Ic: $(\boldsymbol{n}=10) \mathbf{1 0} \%$ carbamide peroxide (Whiteness Perfect, FGM, Joinville, Brazil)

The samples were placed in one arch in vacuum-forming device and 1-mm thick custom trays were fabricated. To prevent the contamination of other surfaces of samples from bleaching agent, the bleaching solution with approximately thickness of $1 \mathrm{~mm}$ was applied uniformly only on the buccal and palatal surface of custom fabricated trays. After daily bleaching procedure, samples were cleaned under running water for 1 minute, dried, and then stored in distilled water till the next application. The gel was placed on the restored buccal and palatal surfaces for 6 to 8 hours daily for 1 week.

Group II. Nanohybrid composite resin $(\boldsymbol{n}=\mathbf{3 0})$ : The composite resin (Herculite Precis, Kerr, California, United States) was inserted (increments) with Teflon coated instrument (GDC) into the prepared cavities and light-cured for 30 second. Finishing and polishing of cavities was done with Sof-Lex discs (3M ESPE).

The samples were coated with two coats of cosmetic nail polish $2 \mathrm{~mm}$ short of all the margins of each restoration before applying the bleaching agents. After that the root apices of 20 samples were mounted in acrylic resin and 10 samples for home bleaching were mounted in one arch \& teeth were stored in distal water till further experiment.

Group II was further subdivided into 3 subgroups, containing 10 samples each.

Subgroup Ila: $(n=10) 35 \%$ hydrogen peroxide (Pola Office, SDI)

Teeth were cleaned with a flour-based pumice. The contents of the syringe were carefully extruded into the pot and immediately mixed using a brush applicator until homogeneous gel was achieved. A thick layer of gel was applied to all the samples and left for 8 minutes. After that buccal and palatal surfaces were washed with water and air dried.

Subgroup IIb: $(n=10) 35 \%$ hydrogen peroxide with calcium (Whiteness HP Blue FGM)

Teeth were cleaned with a flour-based pumice. To prepare the homogeneous gel, connecting the two syringes each other and plungers were pushed four times on each side. This homogenous mix was performed into one syringe, and applied a thick layer ( 0.5 to $1 \mathrm{~mm}$ ) on the surface of samples and left for 40 minutes. With a disposable micro applicator the gel was moved on the samples frequently in every 5 to 10 minutes to release oxygen bubbles generated and renew contact of the gel with the tooth structure. In the end of the treatment, the samples were washed with plenty of water and polished with polishing paste.

Subgroup IIc: $(n=10) 10 \%$ carbamide peroxide (Whiteness Perfect, FGM)

The samples were placed in one arch in vacuum-forming device and $1 \mathrm{~mm}$ thick custom trays were fabricated. To prevent the contamination of other surfaces of samples from bleaching agent, the bleaching solution approximately thickness of $1 \mathrm{~mm}$ was applied uniformly only on the buccal and palatal surface of custom fabricated trays. After daily bleaching procedure, samples were cleaned under running water for 1 minute, dried and then stored in distilled water till the next application. The gel was placed on the restored buccal and palatal surfaces for 6 to 8 hours daily for 1 week.

\section{Group III. Control Group ( $=20)$}

Subgroup IIIa: Negative control $(\boldsymbol{n}=10)$ class V cavities were prepared on buccal and palatal surfaces and restored with microfilled and nanohybrid composite resins. All the samples were fully coated with nail varnish.

Subgroup IIIb: Positive control $(\boldsymbol{n}=10)$ class V cavities were prepared on buccal and palatal surfaces and not restored with any composite resin.

After that the root apices of 20 samples were mounted in acrylic resin and teeth were stored in distal water till further experiment.

All the cavities filled with composite were isolated with two coats of cosmetic nail polish $2 \mathrm{~mm}$ short of all the margins of each restoration before applying the bleaching agents.

\section{Immersion of Samples into Dye Solution}

All the samples were immersed in a contrast solution in $1 \%$ of rhodamine B fluorescent dye (Ases Chemical Works, Jodhpur, India) according to group separately for 24 hours. Finally, the samples were rinsed with distilled water and sectioned buccolingually through the center of the restorations using double-sided diamond cutting disc and right side of sectioned teeth were evaluated for marginal microleakage at both buccal and palatal surfaces.

\section{Evaluation of Marginal Microleakage}

The sectioned samples were examined to check the extent of dye penetration using Confocal Laser Scanning microscope (Nikon's CLSM model A1R) and oil immersion objectives in conjunction with a green filter (wavelength $546 \mathrm{~mm}$ ) along the tooth/restoration interphase and the depth of dye penetration was scored (-Figs. 3-5).

\section{The Scoring Criteria}

\begin{tabular}{|l|l|}
\hline Score & Description \\
\hline 1 & No dye penetration \\
\hline 2 & $\begin{array}{l}\text { Dye penetration not more than half of the } \\
\text { occlusal or gingival wall }\end{array}$ \\
\hline 3 & $\begin{array}{l}\text { Dye penetration more than half of the occlusal or } \\
\text { gingival wall }\end{array}$ \\
\hline 4 & Dye penetration along axial wall \\
\hline
\end{tabular}

\section{Results}

The present in vitro study was designed to evaluate the effects of different bleaching agents (Pola Office, Whiteness HP Blue, and Whiteness Perfect) on marginal integrity of two different restorative resins (microfilled and nanohybrid composite) materials at occlusal and gingival levels by dye penetration method under confocal laser scanning microscope. 


\section{Group III Control Group}
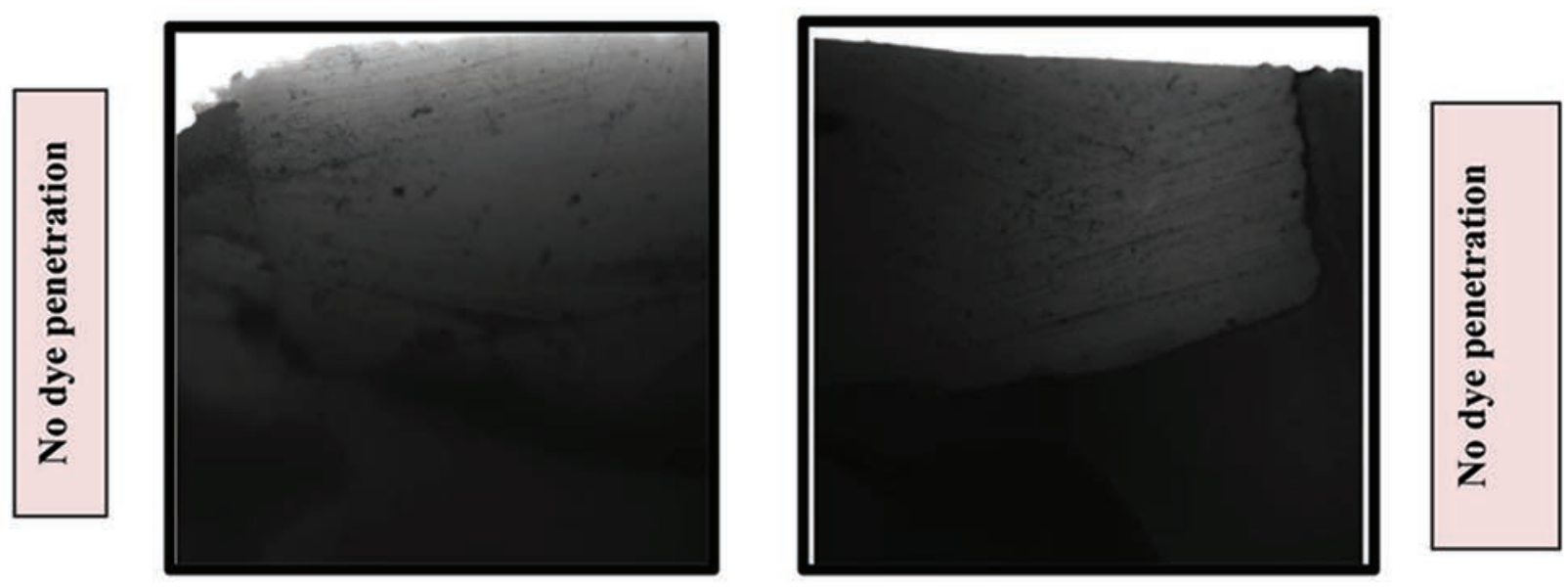

GpIIIa (Negative control) score 3 (Occlusal surface)

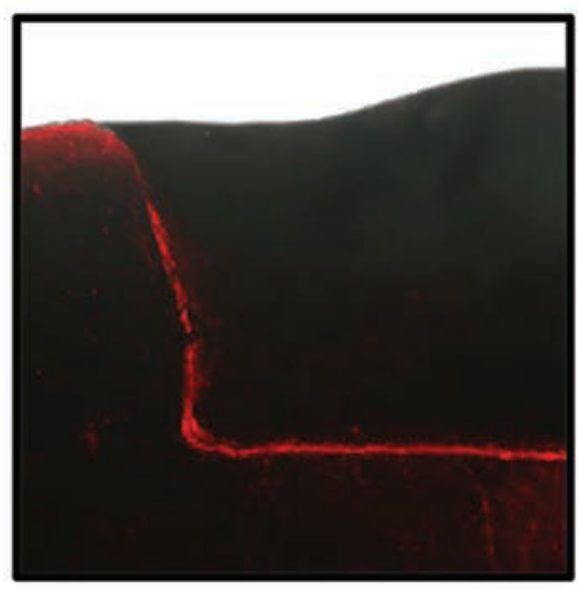

GpIIIb (Positive control) score 3 (Occlusal surface)

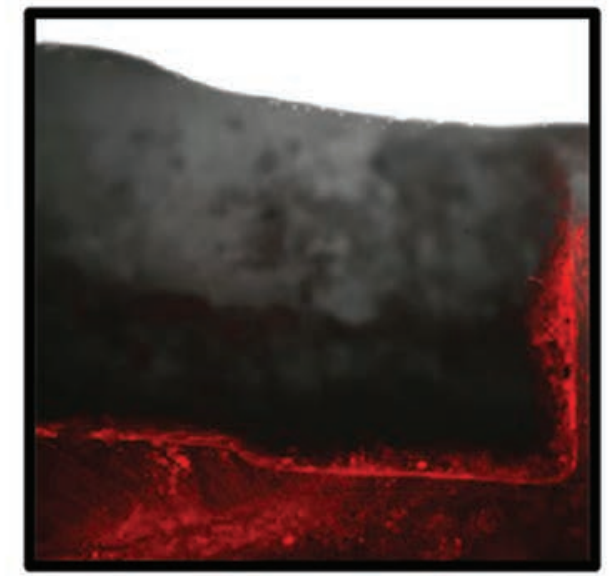

GpIIIa (Negative control) score 3 (Gingival surface)

\section{GpIIIb (Positive control) score 3 (Gingival surface)}

Fig. 3 Confocal images of control groups for both composite resins molars.

Among the composite resin tested, the material group 1 microfilled composite (Heliomolar) exhibited least dye penetration with subgroup1a (Pola Office) and subgroup 1b (Whiteness HP Blue) at occlusal surface but showed less microleakage with subgroup $1 \mathrm{~b}$ at gingival level than other subgroups.Group 2 nanohybrid composite (Herculite Precis) exhibited least dye penetration with subgroup 2a (Pola Office) at both occlusal and gingival levels. Group1 (microfilled composite), Group 2 (nanohybrid composite) showed maximum dye penetration when bleached with whiteness perfect bleaching agent, that is, in subgroup $1 \mathrm{c}$ and subgroup $2 \mathrm{c}$ at both occlusal and gingival levels (-Table $\mathbf{1}$; - Fig. 6 ).

Comparison of subgroup (Pola Office, Whiteness HP Blue, Whiteness Perfect) with in the same group (microfilled and nanohybrid composite resin) at different levels.

Group 1 (microfilled composite resin) at occlusal levels Subgroup Ib < Subgroup Ia < Subgroup Ic

Group 1 (microfilled composite resin) at gingival levels Subgroup Ib < Subgroup Ia < Subgroup Ic

Group 2 (nanohybrid composite resin) at occlusal levels Subgroup Ila < Subgroup IIb < Subgroup IIc 

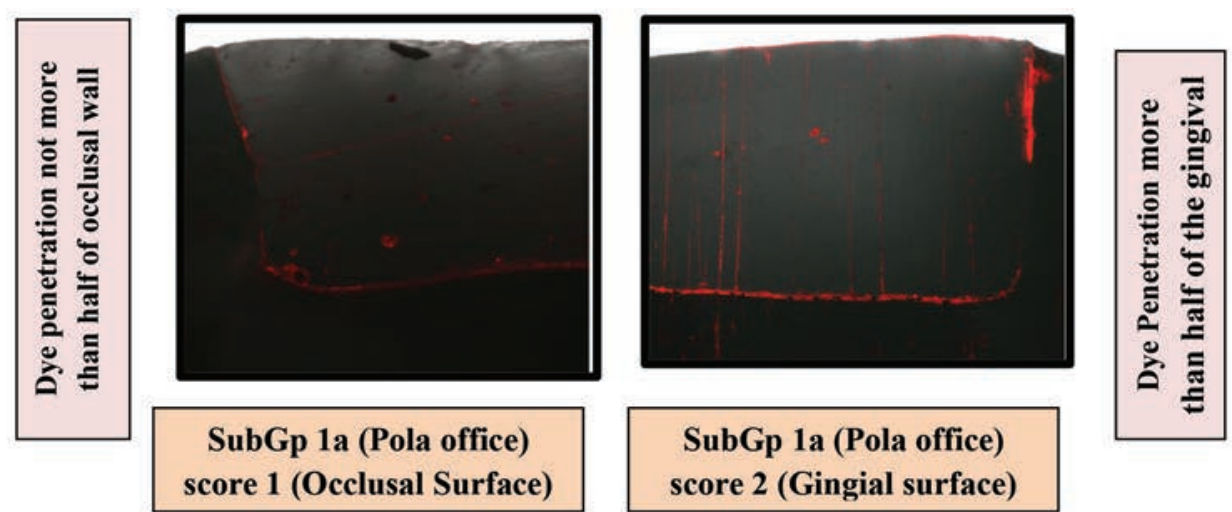

\section{SubGp 1a (Pola office) score 1 (Occlusal Surface)}
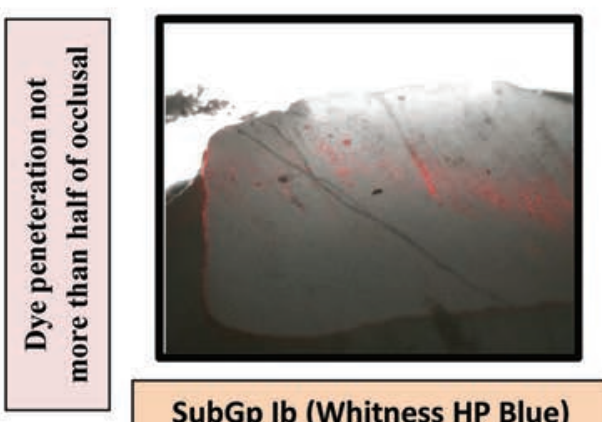

SubGp Ib (Whitness HP Blue) score 1 (Occlusal surface)

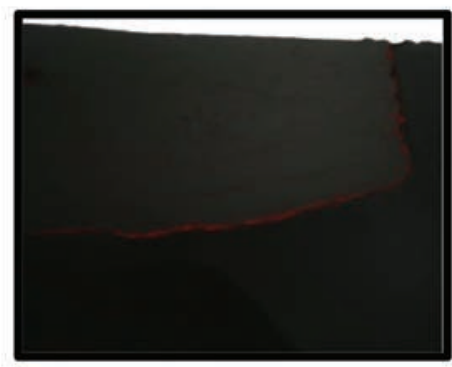

SubGp Ib (Whitness HP Blue)

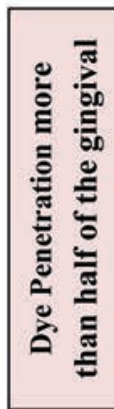
score 2 (Gingical surface)
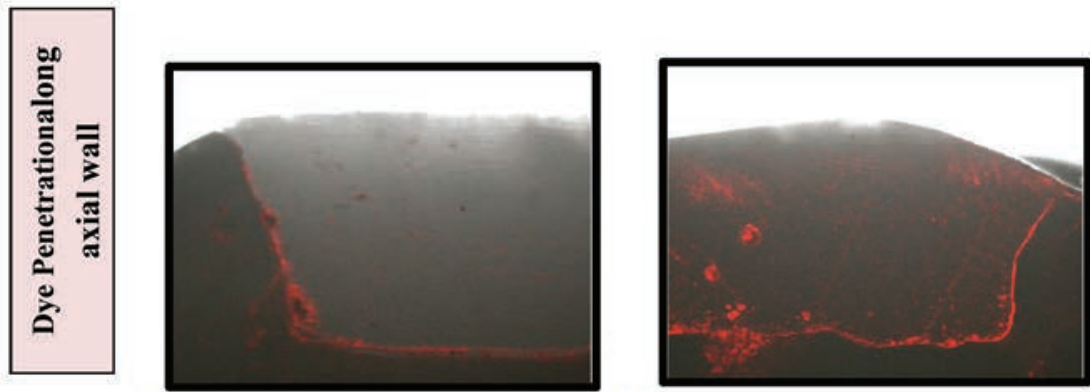

SubGp Ic (Whitness Perfect) score 3 (Occlusal surface)

SubGp Ic (Whitness perfect) score 3 (Gingival surface)

Fig. 4 Confocal images of group I (microfilled composite) with three different bleaching agents.

Group 2 (nanohybrid composite resin) at gingival levels Subgroup IIa < Subgroup IIb < Subgroup IIc

When multiple comparisons of mean dye penetration of different composite resin materials and different bleaching agents were done, there was a significant difference observed between different groups $(p<0.01)$ except in case of subgroups 1, 2a (Pola Office) and subgroups 1, 2b (Whiteness HP Blue) and there was no significant difference observed between different groups at gingival surfaces, that is, $p>0.05$ except when subgroup $1 \mathrm{~b}$ (Whiteness HP Blue) was compared with subgroup 1c (Whiteness Perfect) which gave significant $\mathrm{p}$ value, that is, $\mathrm{P}<005$ ( - Table 2 ).

No significant values were observed at occlusal levels for subgroup $1 \mathrm{a}(0.89)$ and subgroup $2 \mathrm{~b}(0.68)$. So the effects of both bleaching agents, that is, Pola Office and Whiteness HP Blue was same on both the composite used in this study (-Table $\mathbf{3}$ ).
When all composite resin groups were compared with all the bleaching agents, at occlusal and gingival levels lowest marginal leakage scores were obtained with the group I (microfilled composite) resin bleached with Whiteness HP Blue and in (nanohybrid composite) lowest microleakage scores were obtained with Pola Office bleach.

\section{Discussion}

One of the main reasons that patients seek esthetic dental treatment is the real or perceived discoloration of anterior teeth. The restorative filling materials used in dentistry require long-term durability to survive in the oral cavity.

The chemical processes of tooth bleaching could change the physical and mechanical properties of both dental tissue and composite restorative materials causing microleakage. ${ }^{2}$ 

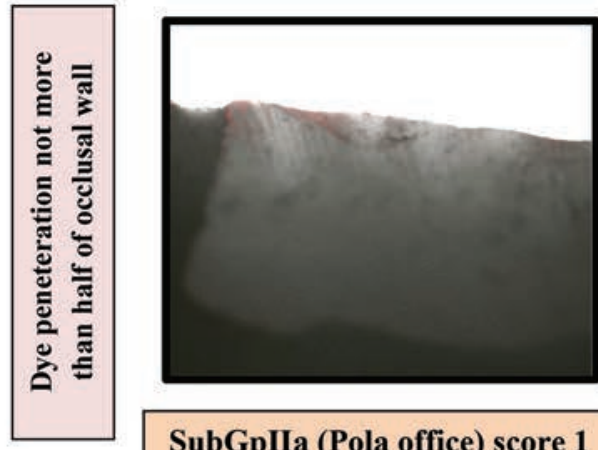

SubGpIIa (Pola office) score 1 (Occlusal surface)
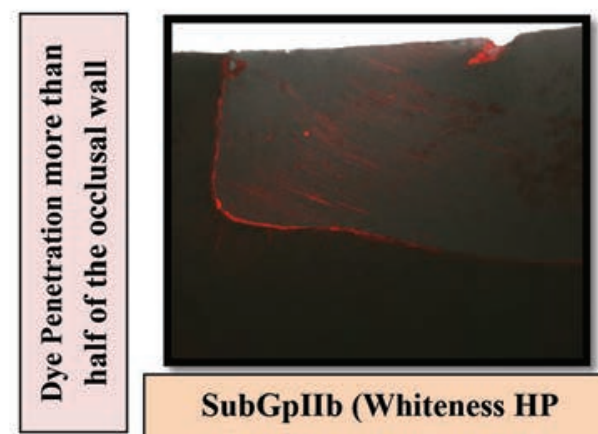

SubGpIIb (Whiteness HP Blue) score 1 (Occlusal surface)

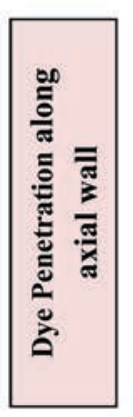

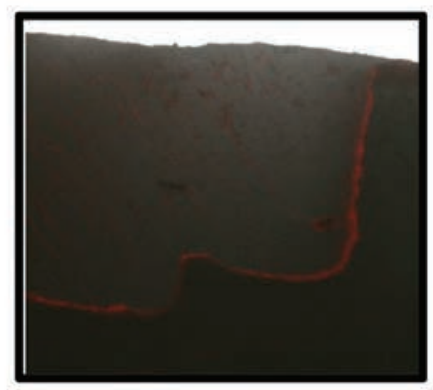

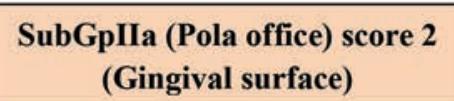

(Gingival surface)
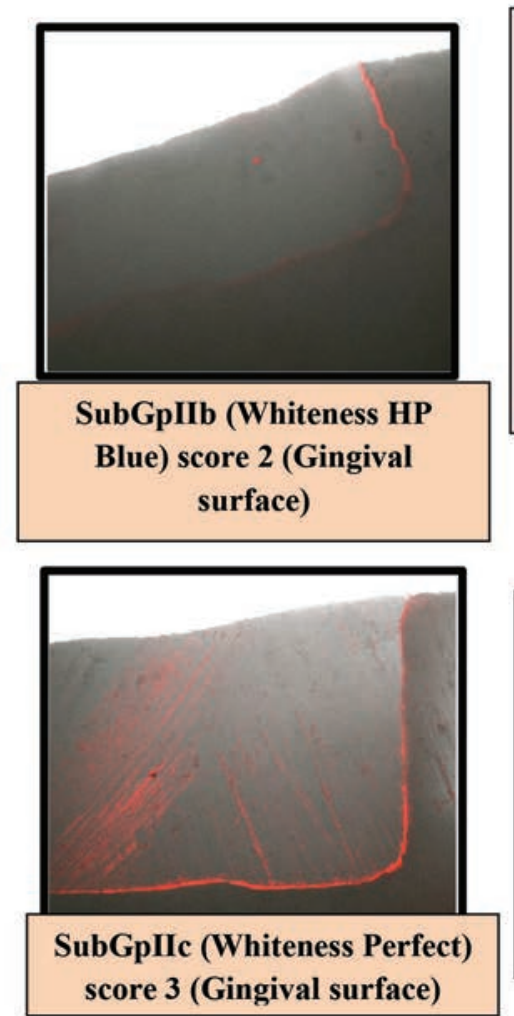
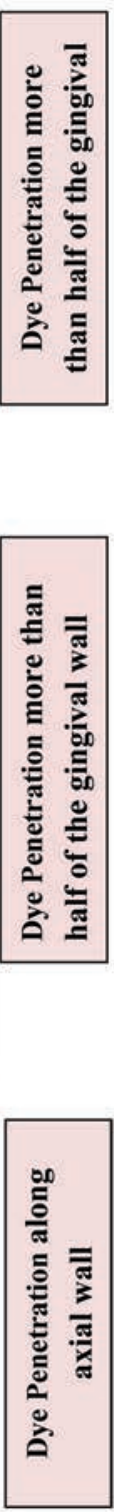

Fig. 5 Confocal images of group II (nanohybrid composite) with three different bleaching agents.

Table 1 The intragroup comparison of mean and standard deviation values of marginal integrity of different composite resin restorations (microfilled and nanohybrid composite) by applying different bleaching agents (Pola Office, Whiteness HP Blue, Whiteness Perfect) at occlusal and gingival levels tested using one-way analysis of variance (ANOVA)

\begin{tabular}{|l|l|l|l|l|l|l|}
\hline Bleaching agents & $\begin{array}{l}\text { Tooth } \\
\text { surfaces }\end{array}$ & \multicolumn{2}{l|}{$\begin{array}{l}\text { Group 1 } \\
\text { microfilled composite } \\
\text { resin (Heliomolar) }\end{array}$} & $\begin{array}{l}\text { Group 2 nanohybrid } \\
\text { composite resin (Herculite } \\
\text { Précis) }\end{array}$ & Status \\
\cline { 3 - 7 } & MEAN & SD & MEAN & SD \\
\hline $\begin{array}{l}\text { Pola Office } \\
\text { (Subgroups 1, 2a) }\end{array}$ & Occlusal & 1.3 & 0.96 & 0.95 & 0.68 & Highly significant \\
\cline { 2 - 7 } & Gingival & 2.2 & 1.00 & 1.85 & 0.93 \\
\hline $\begin{array}{l}\text { Whiteness HP Blue } \\
\text { (Subgroups 1, 2b) }\end{array}$ & Occlusal & 1.3 & 0.65 & 1.3 & 0.80 \\
\hline $\begin{array}{l}\text { Whiteness Perfect } \\
\text { (Subgroups 1, 2c) }\end{array}$ & Gingival & 2.05 & 0.94 & 1.95 & 0.88 \\
\hline
\end{tabular}




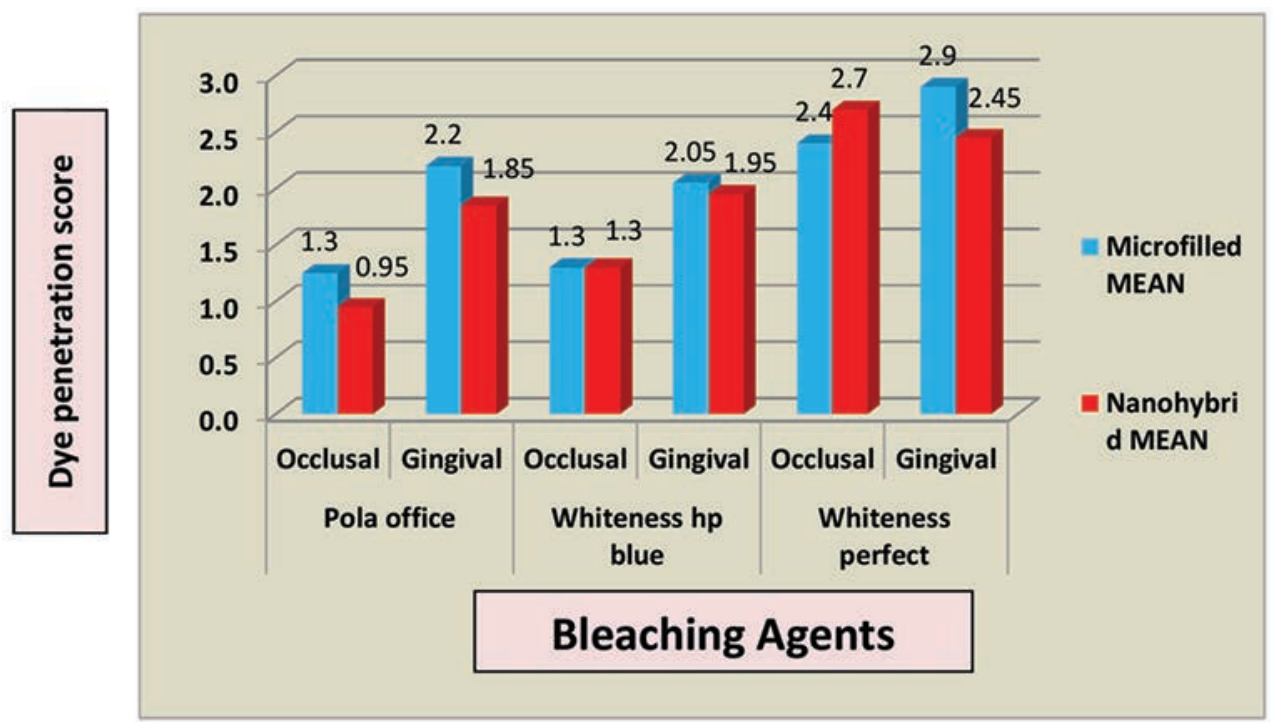

Fig. 6 Graph 1 (bar diagram): Depicts the intragroup comparison of mean values of marginal integrity of different composite resin restorations by applying different bleaching agents at occlusal and gingival levels.

Table 2 The Intergroup comparison of different bleaching agents on microfilled and nanohybrid composite resins on gingival surfaces by using Tukey's HSD test

\begin{tabular}{|c|c|c|c|c|c|}
\hline \multirow{2}{*}{\multicolumn{2}{|c|}{ Comparing pair }} & \multicolumn{2}{|c|}{$\begin{array}{l}\text { Group } 1 \\
\text { microfilled composite resin } \\
\text { (Heliomolar) }\end{array}$} & \multicolumn{2}{|c|}{$\begin{array}{l}\text { Group } 2 \\
\text { nanohybrid composite resin } \\
\text { (Herculite Précis) }\end{array}$} \\
\hline & & $p$-Value & Status & $p$-Value & Status \\
\hline $\begin{array}{l}\text { Pola Office } \\
\text { (subgroups 1, 2a) }\end{array}$ & $\begin{array}{l}\text { Whiteness HP Blue } \\
\text { (subgroups I, Ilb) }\end{array}$ & 0.89 & Nonsignificant & 0.88 & Nonsignificant \\
\hline $\begin{array}{l}\text { Whiteness HP Blue } \\
\text { (subgroups 1, 2b) }\end{array}$ & $\begin{array}{l}\text { Whiteness Perfect } \\
\text { (subgroups I, IIc) }\end{array}$ & 0.01 & Significant & 0.32 & Nonsignificant \\
\hline $\begin{array}{l}\text { Pola Office } \\
\text { (subgroups 1, 2a) }\end{array}$ & $\begin{array}{l}\text { Whiteness Perfect } \\
\text { (subgroups I, Ilc) }\end{array}$ & 0.06 & Nonsignificant & 0.14 & Nonsignificant \\
\hline
\end{tabular}

Table 3 Demonstrate the intergroup comparison of different bleaching agents on microfilled and nanohybrid composite resins on occlusal surfaces by using Tukey's HSD test

\begin{tabular}{|l|l|l|l|l|l|}
\hline \multicolumn{2}{|l|}{ Comparing pair } & \multicolumn{2}{l|}{$\begin{array}{l}\text { Group 1 } \\
\text { microfilled composite resin } \\
\text { (Heliomolar) }\end{array}$} & \multicolumn{2}{l|}{$\begin{array}{l}\text { Group } 2 \\
\text { nanohybrid composite resin } \\
\text { (Herculite Précis) }\end{array}$} \\
\cline { 3 - 6 } & $p$-Value & Status & -Value & Status \\
\hline $\begin{array}{l}\text { Pola Office } \\
\text { (subgroups 1, 2a) }\end{array}$ & $\begin{array}{l}\text { Whiteness HP Blue } \\
\text { (subgroups 1, 2b) }\end{array}$ & 0.89 & Nonsignificant & 0.68 & Nonsignificant \\
\hline $\begin{array}{l}\text { Whiteness HP Blue } \\
\text { (subgroups 1, 2b) }\end{array}$ & $\begin{array}{l}\text { Whiteness Perfect } \\
\text { (subgroups 1, 2c) }\end{array}$ & 0.001 & Significant & 0.001 & Significant \\
\hline $\begin{array}{l}\text { Pola Office } \\
\text { (subgroups 1, 2a) }\end{array}$ & $\begin{array}{l}\text { Whiteness perfect } \\
\text { (subgroups 1, 2c) }\end{array}$ & 0.001 & Significant & 0.001 & Significant \\
\hline
\end{tabular}

During polymerization shrinkage, stresses are generated within the restoration and at the margins, and if these stresses exceed the bond strength, gap formation, and microleakage may occur at the tooth restoration interface. Following factors are involved in polymerization stresses like cavity configuration or C-factor (defined as the ratio of the bonded to the unbonded surface area), cavity size, the technique used to fill the composite resin in the cavity, and the mechanical properties of composite resin itself. ${ }^{1}$ In the current study, efforts were made to maintain all these variables at the same level for both groups. To keep C-factor at the same level, all the class $\mathrm{V}$ cavities were standardized with similar sizes and shapes.

Thus the aim of present study was to evaluate marginal integrity at tooth/composite restoration interface after application of bleaching agents. 


\section{Interpretation and Observation}

In the present study, the results of all samples showed some amount of dye penetration except the negative control group in which no dye penetrated as it was totally sealed with nail varnish.

The adhesive interface is the most susceptible to degradation; exposure to bleaching agents may affect the longevity of the restoration. Although adhesive systems have a higher amount of matrix than resin composites, these changes would be greater and the interfaces are more susceptible to adhesive failure, and it results in marginal destruction. ${ }^{6}$

Crim demonstrated a carbamide peroxide agent adversely affected the marginal sealing ability of both enamel and dentinal of a previously placed resin composite. ${ }^{7}$

Jacob and Kumar reveled postoperative bleaching with $10 \%$ carbamide peroxide may alter the marginal leakage of composite resin restoration, but amalgam restoration are not affected adversely in vitro. ${ }^{8}$

Ulukapi et al revealed that bleaching with $35 \%$ hydrogen peroxide or 10 to $16 \%$ carbamide peroxide adversely affected the marginal seal of resin composite. ${ }^{9}$

It has been shown that hydrogen peroxide could affect the organic and inorganic components of dentin, causing the denaturation of proteins. These morphological changes could also reduce the performance of resin bonded restorations. ${ }^{10}$

Berger et al showed various concentration of hydrogen peroxide penetration occurs due to its low molecular weight and ability to denature proteins, which increases the ionic movement through the dental hard structure and also due to polymerization shrinkage which possibly changes the adhesive interface of enamel and restorations. ${ }^{11}$

It was observed that all bleaching agents caused greater microleakage at gingival margins in comparison to occlusal margins. This is in agreement with reports that hydrogen peroxide can cause degradation of organic components, as well as demineralization of hard tissues. The cementum in close proximity to the CEJ is more organic and thin; therefore, it is easily affected by chemicals such as bleaching agents. ${ }^{12}$

Bektas et al compared microleakage in samples of gingival margins; it was observed that all bleaching agents tested caused greater microleakage in proportion to occlusal margins. ${ }^{12}$

However, all groups showed greater infiltration at the margins located in dentin than in enamel. The reason could be the difference in composition of enamel and dentin. Dentin contains less mineral and more organic matrix, which might easily be affected by bleaching agents. ${ }^{10}$

It is important to know the $\mathrm{pH}$ value of dental bleaching agents because it has a strong effect on hydrogen peroxide chemistry

According to Malkondu and others, in the $\mathrm{pH}$ range of 9.5 to 10.8 , the ionization of buffered hydrogen peroxide produces more perhydroxyl free radicals, resulting in $50 \%$ greater bleaching effects in the same period compared with other $\mathrm{pH}$ levels. ${ }^{3}$

However, more severe changes in the tooth enamel topography were observed when samples were exposed to low-pH products. $^{3}$
Tames et al used a solution of $10 \%$ carbamide peroxide and stated that the $\mathrm{pH}$ may influence the action of bleaching agents onto hard tissues. ${ }^{2}$

According to Sa et al, their study indicated that bleaching agents with low $\mathrm{pH}$ values could induce alterations of enamel surfaces under in vitro situation. ${ }^{13}$

These studies are in favor of the present study. In results of subgroups Ia and IIa (Pola Office), even though the pH is low and application time is less but it still results in destruction of marginal integrity.

The microparticle resins are more susceptible to the adverse effects caused by bleaching agents due to their higher concentration of organic matrix. The softening of composite resins due to the bleaching agent's action is, according to the authors, difficult to explain since no component of the bleaching agent used in their study features parameters of solubility that can interact with Bis-GMA. The reactions caused by free radicals are not specific and may modify various organic structures. Therefore, when a bleaching treatment is performed the enamel organic matrix may be broken and damaged. This would lead to a higher enamel permeability and lower resistance to fracture propagation. These structural alterations induce modifications of the enamel's mechanical properties. ${ }^{2}$

Because Pola Office and Whiteness HP Blue have 35\% hydrogen peroxide and Whiteness Perfect has 10\% carbamide peroxide, Pola Office was applied in three applications of 8 minute each, Whiteness HP Blue was applied for 40 minutes, and Whiteness Perfect (bleaching agent) was applied 6 to 8 hours daily for 1 week on samples.

So whiteness perfect subgroups I and IIc showed worst results with both restorative resins as the time of bleaching agents was more than Pola Office and whiteness HP Blue gel.

Barkhordar et al stated that at home bleaching had a minimum effect on the marginal seal of the existing resin restoration within the first 2 days and a significant effect after 4 to 7 days of bleaching, and concluded that the microleakage increased with the extent of bleaching time. ${ }^{14}$

Bistey et al found that the alterations in enamel were directly proportional to the peroxide concentration and treatment time according to the FT-IR spectroscopy. ${ }^{15}$

According to Sartori et al bleaching agents can cause the loss of calcium and phosphorus from dental structure. Thus, microstructural changes occur in enamel up to a depth of 50 $\mu \mathrm{m} .^{6}$

In present study the subgroup Ib (Whiteness HP Blue) has calcium in its composition.

But subgroup I and IIb (Whiteness HP Blue) did not show any significant results for preservation of marginal integrity of tooth/restoration interface.

In the present study the time period of application of bleaching agents is different, so it was a little difficult to conclude that the destruction of marginal integrity shown by two different reins after bleaching agents. So, in future the experiments should be done on effects of single bleaching agent with different concentrations on different aesthetic restorations. 


\section{Conclusion}

Under the limitations of the current in vitro study, following conclusions can be drawn:

In this in vitro study the treatment with all three bleaching agents (Pola Office, Whiteness HP Blue, Whiteness Perfect) showed destruction of marginal integrity of two different restorative resin materials (microfilled and nanohybrid composite resins) at occlusal and gingival levels.

Among composite resins:

- Group I (microfilled composite) exhibited the lowest mean dye penetration with bleaching agent (Pola Office) as compared with the other bleaching agents.

- Group II (nanohybrid composite) exhibited the lowest mean dye penetration with bleaching agent (Whiteness HP Blue) as compared with other bleaching agents.

- Groups I and II showed highest penetration with bleaching agent (Whiteness Perfect) as compared with other bleaching agents.

- Pola Office bleaching agent showed maximum microleakage even though it is more acidic in short duration.

- Whiteness Perfect (home bleaching) due to more application time showed maximum dye penetration at tooth restoration interface

- (Whiteness HP Blue) containing calcium has no effect in preservation of marginal integrity.

- The effect of bleaching agents was more at gingival levels in comparison to occlusal levels.

\section{Conflict of Interest}

None declared.

\section{References}

1 Fatima N, Mohiuddin S, Iqbal W. Effect on micro-leakage of composite restoration with two different adhesives after bleaching. Smile Dental Journal 2015;10(1):28-32

2 Dutra RA, Branco JRT, Alvim HH, Poletto LTA, Albuquerque RC. Effect of hydrogen peroxide topical application on the enamel and composite resin surfaces and interface. Indian. J Dent Res 2009;20(1):65-70
3 Abe AT, Youssef MN, Turbino ML. Effect of bleaching agents on the nanohardness of tooth enamel, composite resin, and the tooth-restoration interface. Oper Dent 2016;41(1):44-52

4 Briso AL, Tuñas IT, de Almeida LC, Rahal V, Ambrosano GM. Effects of five carbamide peroxide bleaching gels on composite resin microhardness. Acta Odontol Latinoam 2010;23(1):27-31

5 Villalta P, Lu H, Okte Z, Garcia-Godoy F, Powers JM. Effects of staining and bleaching on color change of dental composite resins. J Prosthet Dent 2006;95(2):137-142

6 Sartori N, Junior SM, Filho AM, Arcari GM. Effect of dental bleaching on the microleakage of class $\mathrm{V}$ composite restorations. Revista Odonto Ciência 2009;24(3):279-282

7 Rashwan AT, El-Sharkawey MM, Kamar AA, Abdel-Fattah MM. Effect of two different bleaching concentrations on microleakage and microhardness of tooth-colored restorations (an in vitro study). AlexandriaDent J 2016; (41):122-130

8 Jacob AS, Kumar NMD. Effect of pre and post-operative bleaching on microleakage of amalgam and composite restoration using 10\% carbamide peroxide - an in vitro study. J Conserv Dent 2007;10(1):33-37

9 Ulukapi H, Benderli Y, Ulukapi I. Effect of pre- and postoperative bleaching on marginal leakage of amalgam and composite restorations. Quintessence Int 2003;34(7):505-508

10 Yu H, Li Q, Attin T, Wang Y. Protective effect of resin coating on the microleakage of Class $\mathrm{V}$ restorations following treatment with carbamide peroxide in vitro. Oper Dent 2010;35(6):634-640

11 Berger SB, Tabchoury CP, Ambrosano GM, Giannini M. Hydrogen peroxide penetration into the pulp chamber and dental permeability after bleaching. Gen Dent 2013;61(3):e21-e25

12 Bektas ÖÖ, Eren D, Akin GG, Sag BU, Ozcan M. Microleakage effect on class $\mathrm{V}$ composite restorations with two adhesive systems using different bleaching methods. Acta Odontol Scand 2013;71(3-4):1000-1007

13 Sa Y, Chen D, Liu Y, et al. Effects of two in-office bleaching agents with different $\mathrm{pH}$ values on enamel surface structure and color: an in situ vs. in vitro study. J Dent 2012;40 (Suppl 1):e26-e34

14 Yassen AA, Haridy MF. Leakage of low shrinkage composite with pre and post-restorative tooth bleaching protocols. Egypt Dent J 2014; (60):3171-3179

15 Kleverlaan CJ, Feilzer AJ. Polymerization shrinkage and contraction stress of dental resin composites. Dent Mater 2005;21(12):1150-1157 